\title{
MEASURES OF SECTIONS OF CONVEX BODIES
}

\author{
ALEXANDER KOLDOBSKY
}

\begin{abstract}
This article is a survey of recent results on slicing inequalities for convex bodies. The focus is on the setting of arbitrary measures in place of volume.
\end{abstract}

\section{INTRODUCTION}

The study of volume of sections of convex bodies is a classical direction in convex geometry. It is well developed and has numerous applications; see [G3, K4]. The question of what happens if volume is replaced by an arbitrary measure on a convex body has not been considered until very recently, mostly because it is hard to believe that difficult geometric results can hold in such generality. However, in 2005 Zvavitch $[\mathrm{Zv}]$ proved that the solution to the Busemann-Petty problem, one of the signature problems in convex geometry, remains exactly the same if volume is replaced by an arbitrary measure with continuous density. It has recently been shown [K6, KM, K8, K9, K10, K11] that several partial results on the slicing problem, a major open question in the area, can also be extended to arbitrary measures. For example, it was proved in [K11] that the slicing problem for sections of proportional dimensions has an affirmative answer which can be extended to the setting of arbitrary measures. It is not clear yet whether these results are representative of something bigger, or it is just an isolated event. We let the reader make the judgement.

\section{The SLICING PROBLEM FOR MEASURES}

The slicing problem [Bo1, Bo2, Ba5, MP], asks whether there exists an absolute constant $C$ so that for every origin-symmetric convex body $K$ in $\mathbb{R}^{n}$ of volume 1 there is a hyperplane section of $K$ whose $(n-1)$ dimensional volume is greater than $1 / C$. In other words, does there exist a constant $C$ so that for any $n \in \mathbb{N}$ and any origin-symmetric convex body $K$ in $\mathbb{R}^{n}$

$$
|K|^{\frac{n-1}{n}} \leq C \max _{\xi \in S^{n-1}}\left|K \cap \xi^{\perp}\right|,
$$


where $\xi^{\perp}$ is the central hyperplane in $\mathbb{R}^{n}$ perpendicular to $\xi$, and $|K|$ stands for volume of proper dimension? The best current result $C \leq O\left(n^{1 / 4}\right)$ is due to Klartag [Kla2], who slightly improved an earlier estimate of Bourgain [Bo3]. The answer is known to be affirmative for some special classes of convex bodies, including unconditional convex bodies (as initially observed by Bourgain; see also [MP, J2, BN]), unit balls of subspaces of $L_{p}$ [Ba4, J1, M1], intersection bodies [G3, Th.9.4.11], zonoids, duals of bodies with bounded volume ratio [MP], the Schatten classes $[\mathrm{KMP}], k$-intersection bodies [KPY, K10]. Other partial results on the problem include [Ba3, BKM, DP, Da, GPV, Kla1, KlaK, Pa, EK, BaN]; see the book [BGVV] for details.

Iterating (1) one gets the lower dimensional slicing problem asking whether the inequality

$$
|K|^{\frac{n-k}{n}} \leq C^{k} \max _{H \in G r_{n-k}}|K \cap H|
$$

holds with an absolute constant $C$, where $1 \leq k \leq n-1$ and $G r_{n-k}$ is the Grassmanian of $(n-k)$-dimensional subspaces of $\mathbb{R}^{n}$.

Inequality (2) was proved in [K11] in the case where $k \geq \lambda n, 0<$ $\lambda<1$, with the constant $C=C(\lambda)$ dependent only on $\lambda$. Moreover, this was proved in [K11] for arbitrary measures in place of volume. We consider the following generalization of the slicing problem to arbitrary measures and to sections of arbitrary codimension.

Problem 1. Does there exist an absolute constant $C$ so that for every $n \in \mathbb{N}$, every integer $1 \leq k<n$, every origin-symmetric convex body $K$ in $\mathbb{R}^{n}$, and every measure $\mu$ with non-negative even continuous density $f$ in $\mathbb{R}^{n}$,

$$
\mu(K) \leq C^{k} \max _{H \in G r_{n-k}} \mu(K \cap H)|K|^{k / n} .
$$

Here $\mu(B)=\int_{B} f$ for every compact set $B$ in $\mathbb{R}^{n}$, and $\mu(B \cap H)=$ $\int_{B \cap H} f$ is the result of integration of the restriction of $f$ to $H$ with respect to Lebesgue measure in $H$. The case of volume corresponds to $f \equiv 1$.

In some cases we will write (3) in an equivalent form

$$
\mu(K) \leq C^{k} \frac{n}{n-k} c_{n, k} \max _{H \in G r_{n-k}} \mu(K \cap H)|K|^{k / n},
$$

where $c_{n, k}=\left|B_{2}^{n}\right|^{\frac{n-k}{n}} /\left|B_{2}^{n-k}\right|$, and $B_{2}^{n}$ is the unit Euclidean ball in $\mathbb{R}^{n}$. It is easy to see that $c_{n, k} \in\left(e^{-k / 2}, 1\right)$, and $\frac{n}{n-k} \in\left(1, e^{k}\right)$, so these constants can be incorporated in the constant $C$. 
Surprisingly, many partial results on the original slicing problem can be extended to the setting of arbitrary measures. Inequality (3) holds true in the following cases:

- for arbitrary $n, K, \mu$ and $k \geq \lambda n$, where $\lambda \in(0,1)$, with the constant $C$ dependent only on $\lambda$, [K11];

- for all $n, K, \mu, k$, with $C \leq O(\sqrt{n})$, [K8, K9];

- for intersection bodies $K$ (see definition below), with an absolute constant $C,[\mathrm{~K} 6]$ for $k=1,[\mathrm{KM}]$ for all $k$;

- for the unit balls of $n$-dimensional subspaces of $L_{p}, p>2$, with $C \leq O\left(n^{1 / 2-1 / p}\right),[\mathrm{K} 10]$

- for the unit balls of $n$-dimensional normed spaces that embed in $L_{p}, p \in(-n, 2]$, with $C$ depending only on $p,[\mathrm{~K} 10]$;

- for unconditional convex bodies, with an absolute constant $C$, [K11];

- for duals of convex bodies with bounded volume ratio, with an absolute constant $C,[\mathrm{~K} 11]$;

- for $k=1$ and log-concave measures $\mu$, with $C \leq O\left(n^{1 / 4}\right)$, [KZ].

The proofs of these results are based on stability in volume comparison problems introduced in [K5] and developed in [K6, KM, K7, K8, K9, K10, K13]. Stability reduces Problem 1 to estimating the outer volume ratio distance from a convex body to the classes of generalized intersection bodies. The concept of an intersection body was introduced by Lutwak $[\mathrm{Lu}]$ in connection with the Busemann-Petty problem.

A closed bounded set $K$ in $\mathbb{R}^{n}$ is called a star body if every straight line passing through the origin crosses the boundary of $K$ at exactly two points different from the origin, the origin is an interior point of $K$, and the boundary of $K$ is continuous.

For $1 \leq k \leq n-1$, the classes $\mathcal{B P}_{k}^{n}$ of generalized $k$-intersection bodies in $\mathbb{R}^{n}$ were introduced by Zhang [Z3]. The case $k=1$ represents the original class of intersection bodies $\mathcal{I}_{n}=\mathcal{B P}_{1}^{n}$ of Lutwak [Lu]. We define $\mathcal{B P}_{k}^{n}$ as the closure in the radial metric of radial $k$-sums of finite collections of origin-symmetric ellipsoids (the equivalence of this definition to the original definitions of Lutwak and Zhang was established by Goodey and Weil [GW] for $k=1$ and by Grinberg and Zhang [GrZ] for arbitrary $k$.) Recall that the radial $k$-sum of star bodies $K$ and $L$ in $\mathbb{R}^{n}$ is a new star body $K+{ }_{k} L$ whose radius in every direction $\xi \in S^{n-1}$ is given by

$$
r_{K{ }_{k} L}^{k}(\xi)=r_{K}^{k}(\xi)+r_{L}^{k}(\xi)
$$


The radial metric in the class of origin-symmetric star bodies is defined by

$$
\rho(K, L)=\sup _{\xi \in S^{n-1}}\left|r_{K}(\xi)-r_{L}(\xi)\right| .
$$

The following stability theorem was proved in [K11] (see [K6, KM] for slightly different versions).

Theorem 1. ([K11]) Suppose that $1 \leq k \leq n-1, K$ is a generalized $k$ intersection body in $\mathbb{R}^{n}, f$ is an even continuous non-negative function on $K$, and $\varepsilon>0$. If

$$
\int_{K \cap H} f \leq \varepsilon, \quad \forall H \in G r_{n-k}
$$

then

$$
\int_{K} f \leq \frac{n}{n-k} c_{n, k}|K|^{k / n} \varepsilon .
$$

The constant is the best possible. Recall that $c_{n, k} \in\left(e^{-k / 2}, 1\right)$.

Define the outer volume ratio distance from an origin-symmetric star body $K$ in $\mathbb{R}^{n}$ to the class $\mathcal{B P}_{k}^{n}$ of generalized $k$-intersection bodies by

$$
\text { o.v.r. }\left(K, \mathcal{B P}_{k}^{n}\right)=\inf \left\{\left(\frac{|D|}{|K|}\right)^{1 / n}: K \subset D, D \in \mathcal{B P}_{k}^{n}\right\} \text {. }
$$

Theorem 1 immediately implies a slicing inequality for arbitrary measures and origin-symmetric star bodies.

Corollary 1. Let $K$ be an origin-symmetric star body in $\mathbb{R}^{n}$. Then for any measure $\mu$ with even continuous density on $K$ we have

$$
\left.\mu(K) \leq \text { (o.v.r. }\left(K, \mathcal{B P}_{k}^{n}\right)\right)^{k} \frac{n}{n-k} c_{n, k} \max _{H \in G r_{n-k}} \mu(K \cap H)|K|^{k / n} .
$$

Thus, stability reduces Problem 1 to estimating the outer volume ratio distance from $K$ to the class of generalized $k$-intersection bodies. The results on Problem 1 mentioned above were all obtained by estimating this distance by means of various techniques from the local theory of Banach spaces. For example, the solution to the slicing problem for sections of proportional dimensions follows from an estimate obtained in [KPZ]: for any origin-symmetric convex body $K$ in $\mathbb{R}^{n}$ and any $1 \leq k \leq n-1$,

$$
\text { o.v.r. }\left(K, \mathcal{B P}_{k}^{n}\right) \leq C_{0} \sqrt{\frac{n}{k}}\left(\log \left(\frac{e n}{k}\right)\right)^{3 / 2},
$$


where $C_{0}$ is an absolute constant. The proof of this estimate in [KPZ] is quite involved. It uses covering numbers, Pisier's generalization of Milman's reverse Brunn-Minkowski inequality, properties of intersection bodies. Combining this with Corollary 1, one gets

Theorem 2. ([K11]) If the codimension of sections $k$ satisfies $\lambda n \leq k$ for some $\lambda \in(0,1)$, then for every origin-symmetric convex body $K$ in $\mathbb{R}^{n}$ and every measure $\mu$ with continuous non-negative density in $\mathbb{R}^{n}$,

$$
\mu(K) \leq C^{k}\left(\sqrt{\frac{(1-\log \lambda)^{3}}{\lambda}}\right)^{k} \max _{H \in G r_{n-k}} \mu(K \cap H)|K|^{k / n},
$$

where $C$ is an absolute constant.

For arbitrary $K, \mu$ and $k$ the best result so far is the following $\sqrt{n}$ estimate; see [K8, K9]. By John's theorem, for any origin-symmetric convex body $K$ there exists an ellipsoid $\mathcal{E}$ so that $\frac{1}{\sqrt{n}} \mathcal{E} \subset K \subset \mathcal{E}$. Since every ellipsoid is a generalized $k$-intersection body for every $k$, we get that

$$
\text { o.v.r. }\left(K, \mathcal{B} \mathcal{P}_{k}^{n}\right) \leq \sqrt{n}
$$

By Corollary 1,

$$
\mu(K) \leq n^{k / 2} \frac{n}{n-k} c_{n, k} \max _{H \in G r_{n-k}} \mu(K \cap H)|K|^{k / n} .
$$

Note that the condition that the measure $\mu$ has continuous density is necessary in Problem 1. A discrete version of inequality (3) was very recently established (with a constant depending only on the dimension) in $[\mathrm{AHZ}]$.

\section{The isomorphic Busemann-Petty problem}

In 1956, Busemann and Petty [BP] asked the following question. Let $K, L$ be origin-symmetric convex bodies in $\mathbb{R}^{n}$ such that

$$
\left|K \cap \xi^{\perp}\right| \leq\left|L \cap \xi^{\perp}\right|, \quad \forall \xi \in S^{n-1} .
$$

Does it necessarily follow that $|K| \leq|L|$ ? The problem was solved at the end of the 1990's in a sequence of papers [LR, Ba1, Gi, Bo4, Lu, P, G1, G2, Z1, K1, K2, Z2, GKS]; see [K4, p.3] or [G3, p.343] for the solution and its history. The answer is affirmative if $n \leq 4$, and it is negative if $n \geq 5$.

The lower dimensional Busemann-Petty problem asks the same question for sections of lower dimensions. Suppose that $1 \leq k \leq n-1$, and $K, L$ are origin-symmetric convex bodies in $\mathbb{R}^{n}$ such that

$$
|K \cap H| \leq|L \cap H|, \quad \forall H \in G r_{n-k} .
$$


Does it follow that $|K| \leq|L|$ ? It was proved in [BZ] (see also [K3, $\mathrm{K} 4, \mathrm{RZ}, \mathrm{M} 2]$ for different proofs) that the answer is negative if the dimension of sections $n-k>3$. The problem is still open for two- and three-dimensional sections $(n-k=2,3, n \geq 5)$.

Since the answer to the Busemann-Petty problem is negative in most dimensions, it makes sense to ask whether the inequality for volumes holds up to an absolute constant, namely, does there exist an absolute constant $C$ such that inequalities (6) imply $|K| \leq C|L|$ ? This question is known as the isomorphic Busemann-Petty problem, and in the hyperplane case it is equivalent to the slicing problem; see [MP]. A version of this problem for sections of proportional dimensions was proved in [K12].

Theorem 3. ([K12]) Suppose that $0<\lambda<1, k>\lambda n$, and $K, L$ are origin-symmetric convex bodies in $\mathbb{R}^{n}$ satisfying the inequalities

$$
|K \cap H| \leq|L \cap H|, \quad \forall H \in G r_{n-k} .
$$

Then

$$
|K|^{\frac{n-k}{n}} \leq(C(\lambda))^{k}|L|^{\frac{n-k}{n}}
$$

where $C(\lambda)$ depends only on $\lambda$.

This result implies Theorem 2 in the case of volume. It is not clear, however, whether Theorem 2 can be directly used to prove Theorem 3 .

Zvavitch $[\mathrm{Zv}]$ has found a remarkable generalization of the BusemannPetty problem to arbitrary measures in place of volume. Suppose that $1 \leq k<n, \mu$ is a measure with even continuous density $f$ in $\mathbb{R}^{n}$, and $K$ and $L$ are origin-symmetric convex bodies in $\mathbb{R}^{n}$ so that

$$
\mu\left(K \cap \xi^{\perp}\right) \leq \mu\left(L \cap \xi^{\perp}\right), \quad \forall \xi \in S^{n-1} .
$$

Does it necessarily follow that $\mu(K) \leq \mu(L)$ ? The answer is the same as for volume - affirmative if $n \leq 4$ and negative if $n \geq 5$. An isomorphic version was recently proved in [KZ], namely, for every dimension $n$ inequalities (8) imply $\mu(K) \leq \sqrt{n} \mu(L)$. It is not known whether the constant $\sqrt{n}$ is optimal for arbitrary measures. Also there is no known direct connection between the isomorphic Busemann-Petty problem for arbitrary measures and Problem 1.

\section{Projections of Convex Bodies.}

The projection analog of the Busemann-Petty problem is known as Shephard's problem, posed in 1964 in [Sh]. Denote by $K \mid \xi^{\perp}$ the orthogonal projection of $K$ to $\xi^{\perp}$. Suppose that $K$ and $L$ are origin-symmetric convex bodies in $\mathbb{R}^{n}$ so that $|K| \xi^{\perp}|\leq| L\left|\xi^{\perp}\right|$ for every $\xi \in S^{n-1}$. Does it follow that $|K| \leq|L|$ ? The problem was solved by Petty [Pe] and 
Schneider [Sch], independently, and the answer if affirmative only in dimension 2.

Both solutions use the fact that the answer to Shephard's problem is affirmative in every dimension under the additional assumption that $L$ is a projection body. An origin symmetric convex body $L$ in $\mathbb{R}^{n}$ is called a projection body if there exists another convex body $K$ so that the support function of $L$ in every direction is equal to the volume of the hyperplane projection of $K$ to this direction: for every $\xi \in S^{n-1}$,

$$
h_{L}(\xi)=|K| \xi^{\perp} \mid \text {. }
$$

The support function $h_{L}(\xi)=\max _{x \in L}|(\xi, x)|$ is equal to the dual norm $\|\xi\|_{L^{*}}$, where $L^{*}$ denotes the polar body of $L$.

Separation in Shephard's problem was proved in [K5].

Theorem 4. ([K5]) Suppose that $\varepsilon>0, K$ and $L$ are origin-symmetric convex bodies in $\mathbb{R}^{n}$, and $L$ is a projection body. If $|K| \xi^{\perp}|\leq| L\left|\xi^{\perp}\right|-\varepsilon$ for every $\xi \in S^{n-1}$, then $|K|^{\frac{n-1}{n}} \leq|L|^{\frac{n-1}{n}}-c_{n, 1} \varepsilon$, where $c_{n, 1}$ is the same constant as in Theorem 1; recall that $c_{n, 1}>1 / \sqrt{e}$.

Stability in Shephard's problem turned out to be more difficult, and it was proved in [K13] only up to a logarithmic term and under an additional assumtpion that the body $L$ is isotropic. Recall that a convex body $D$ in $\mathbb{R}^{n}$ is isotropic if $|D|=1$ and $\int_{D}(x, \xi)^{2} d x$ is a constant function of $\xi \in S^{n-1}$. Every convex body has a linear image that is isotropic; see [BGVV].

Theorem 5. ([K13]) Suppose that $\varepsilon>0, K$ and $L$ are origin-symmetric convex bodies in $\mathbb{R}^{n}$, and $L$ is a projection body which is a dilate of an isotropic body. If $|K| \xi^{\perp}|\leq| L\left|\xi^{\perp}\right|+\varepsilon$ for every $\xi \in S^{n-1}$, then $|K|^{\frac{n-1}{n}} \leq|L|^{\frac{n-1}{n}}+C \varepsilon \log ^{2} n$, where $C$ is an absolute constant.

The proof is based on an estimate for the mean width of a convex body obtained by E.Milman [M3].

The projection analog of the slicing problem reads as

$$
|K|^{\frac{n-1}{n}} \geq c \min _{\xi \in S^{n-1}}|K| \xi^{\perp} \mid,
$$

and it was solved by Ball [Ba2], who proved that $c$ may and has to be of the order $1 / \sqrt{n}$.

The possibility of extension of Shephard's problem and related stability and separation results to arbitrary measures is an open question. Also, the lower dimensional Shephard problem was solved by Goodey and Zhang [GZ], but stability and separation for the lower dimensional case have not been established. 
Stability and separation for projections have an interesting application to surface area. If $L$ is a projection body, so is $L+\varepsilon B_{2}^{n}$ for every $\varepsilon>0$. Applying stability in Shephard's problem to this pair of bodies, dividing by $\varepsilon$ and sending $\varepsilon$ to zero, one gets a hyperplane inequality for surface area (see $[\mathrm{K} 7])$ : if $L$ is a projection body, then

$$
S(L) \geq c \min _{\xi \in S^{n-1}} S\left(L \mid \xi^{\perp}\right)|L|^{\frac{1}{n}} .
$$

On the other hand, applying separation to any projection body $L$ which is a dilate of a body in isotropic position (see [K13])

$$
S(L) \leq C \log ^{2} n \max _{\xi \in S^{n-1}} S\left(L \mid \xi^{\perp}\right)|L|^{\frac{1}{n}}
$$

Here $c$ and $C$ are absolute constants, and $S(L)$ is surface area.

\section{REFERENCES}

[AHZ] M. Alexander, M. Henk And A. Zvavitch, A discrete version of Koldobsky's slicing inequality, arXiv:1511.02702.

[Ba1] K. BALL, Some remarks on the geometry of convex sets, Geometric aspects of functional analysis (1986/87), Lecture Notes in Math. 1317, SpringerVerlag, Berlin-Heidelberg-New York, 1988, 224-231.

[Ba2] K. BALl, Shadows of convex bodies, Trans. Amer. Math. Soc. 327 (1991), 891-901.

[Ba3] K. BALL, Logarithmically concave functions and sections of convex sets in $\mathbb{R}^{n}$, Studia Math. 88 (1988), 69-84.

[Ba4] K. BALL, Normed spaces with a weak-Gordon-Lewis property, Functional analysis (Austin, TX, 1987/1989), 36-47, Lecture Notes in Math., 1470, Springer, Berlin, 1991.

[Ba5] K. BALL, Isometric problems in $\ell_{p}$ and sections of convex sets, Ph.D. dissertation, Trinity College, Cambridge (1986).

[BaN] K. Ball and V. H. NGuyen, Entropy jumps for isotropic log-concave random vectors and spectral gap, Studia Math. 213 (2012), 81-96.

[BN] S. BobKov AND F. NAZARov, On convex bodies and log-concave probability measures with unconditional basis, Geometric aspects of functional analysis (Milman-Schechtman, eds), Lecture Notes in Math. 1807 (2003), 53-69.

[Bo1] J. Bourgain, On high-dimensional maximal functions associated to convex bodies, Amer. J. Math. 108 (1986), 1467-1476.

[Bo2] J. Bourgain, Geometry of Banach spaces and harmonic analysis, Proceedings of the International Congress of Mathematicians (Berkeley, CA, 1986), Amer. Math. Soc., Providence, RI, 1987, 871-878.

[Bo3] J. Bourgain, On the distribution of polynomials on high-dimensional convex sets, Lecture Notes in Math. 1469 (1991), 127-137.

[Bo4] J. Bourgain, On the Busemann-Petty problem for perturbations of the ball, Geom. Funct. Anal. 1 (1991), 1-13.

[BKM] J. Bourgain, B. Klartag, V. Milman, Symmetrization and isotropic constants of convex bodies, Geometric Aspects of Functional Analysis, Lecture Notes in Math. 1850 (2004), 101-116. 
[BZ] J. Bourgain, Gaoyong Zhang, On a generalization of the BusemannPetty problem, Convex geometric analysis (Berkeley, CA, 1996), 65-76, Math. Sci. Res. Inst. Publ., 34, Cambridge Univ. Press, Cambridge, 1999.

[BGVV] S. Brazitikos, A. Giannopoulos, P. Valettas and B. Vritsiou, Geometry of isotropic log-concave measures, Amer. Math. Soc., Providence, RI, 2014.

[BP] H. Busemann and C. M. Petty, Problems on convex bodies, Math. Scand. 4 (1956), 88-94.

[DP] N. DAFnis And G. PAOuRIS, Small ball probability estimates, $\psi_{2}$-behavior and the hyperplane conjecture, J. Funct. Anal. 258 (2010), 19331964.

[Da] S. DAR, Remarks on Bourgain's problem on slicing of convex bodies, Operator theory, Advances and Applications 77 (1995), 61-66.

[EK] R. Eldan And B. KlartaG, Approximately gaussian marginals and the hyperplane conjecture, Proceedings of the Workshop on "Concentration, Functional Inequalities and Isoperimetry", Contemp. Math. 545 (2011), 55-68.

[G1] R. J. Gardner, Intersection bodies and the Busemann-Petty problem, Trans. Amer. Math. Soc. 342 (1994), 435-445.

[G2] R. J. Gardner, A positive answer to the Busemann-Petty problem in three dimensions, Annals of Math. 140 (1994), 435-447.

[G3] R. J. Gardner, Geometric tomography, Second edition, Cambridge University Press, Cambridge, 2006, 492 p.

[GKS] R.J. Gardner, A. Koldobsky, Th. Schlumprecht, An analytic solution to the Busemann-Petty problem on sections of convex bodies, Annals of Math. 149 (1999), 691-703 .

[Gi] A. Giannopoulos, A note on a problem of H. Busemann and C. M. Petty concerning sections of symmetric convex bodies, Mathematika 37 (1990), 239244.

[GPV] A. Giannopoulos, G. Paouris and B. Vritsiou, A remark on the slicing problem, J. Funct. Anal. 262 (2012), 1062-1086.

[GW] P. Goodey AND W. WeIL, Intersection bodies and ellipsoids, Mathematika 42 (1958), 295-304.

[GZ] P. Goodey And GaOyong Zhang, Inequalities between projection functions of convex bodies, Amer. J. Math. 120 (1998) 345-367.

[GrZ] E. Grinberg and Gaoyong Zhang, Convolutions, transforms, and convex bodies, Proc. London Math. Soc. 78 (1999), 77-115.

[J1] M. Junge, On the hyperplane conjecture for quotient spaces of $L_{p}$, Forum Math. 6 (1994), 617-635.

[J2] M. Junge, Proportional subspaces of spaces with unconditional basis have good volume properties, Geometric aspects of functional analysis (Israel Seminar, 1992-1994), 121-129, Oper. Theory Adv. Appl., 77, Birkhauser, Basel, 1995.

[Kla1] B. Klartag, An isomorphic version of the slicing problem, J. Funct. Anal. 218 (2005), 372-394.

[Kla2] B. KlartaG, On convex perturbations with a bounded isotropic constant, Geom. Funct. Anal. 16 (2006), 1274-1290.

[KlaK] B. Klartag and G. Kozma, On the hyperplane conjecture for random convex sets, Israel J. Math. 170 (2009), 253-268. 
[K1] A. Koldobsky, Intersection bodies, positive definite distributions and the Busemann-Petty problem, Amer. J. Math. 120 (1998), 827-840.

[K2] A. Koldobsky, Intersection bodies in $\mathbb{R}^{4}$, Adv. Math. 136 (1998), 1-14.

[K3] A. Koldobsky, A functional analytic approach to intersection bodies, Geom. Funct. Anal. 10 (2000), 1507-1526.

[K4] A. Koldobsky, Fourier analysis in convex geometry, Amer. Math. Soc., Providence RI, 2005, 170 p.

[K5] A. Koldobsky Stability in the Busemann-Petty and Shephard problems, Adv. Math. 228 (2011), 2145-2161.

[K6] A. Koldobsky, A hyperplane inequality for measures of convex bodies in $\mathbb{R}^{n}, n \leq 4$, Discrete Comput. Geom. 47 (2012), 538-547.

[K7] A. Koldobsky, Stability and separation in volume comparison problems, Math. Model. Nat. Phenom. 8 (2012), 159-169.

[K8] A. Koldobsky, A $\sqrt{n}$ estimate for measures of hyperplane sections of convex bodies, Adv. Math. 254 (2014), 33-40.

[K9] A. Koldobsky, Estimates for measures of sections of convex bodies, GAFA Seminar Volume, B.Klartag and E.Milman, editors, Lect. Notes in Math. 2116 (2014), 261-271.

[K10] A. Koldobsky, Slicing inequalities for subspaces of $L_{p}$, Proc. Amer. Math. Soc., to appear; arXiv:1310.8102

[K11] A. Koldobsky, Slicing inequalities for measures of convex bodies, Adv. Math. 283 (2015), 473-488.

[K12] A. Koldobsky, Isomorphic Busemann-Petty problem for sections of proportional dimensions, Adv. in Appl. Math. 71 (2015), 138-145.

[K13] A. Koldobsky, Stability inequalities for projections of convex bodies, submitted; arXiv:1506.04290

[KM] A. Koldobsky AND DAN Ma, Stability and slicing inequalities for intersection bodies, Geom. Dedicata 162 (2013), 325-335.

[KPY] A. Koldobsky, A. PAjor And V. Yaskin, Inequalities of the KahaneKhinchin type and sections of $L_{p}$-balls, Studia Math. 184 (2008), 217-231.

[KPZ] A. Koldobsky, G. PaOuris and M. Zymonopoulou, Isomorphic properties of intersection bodies, J. Funct. Anal. 261 (2011), 2697-2716.

[KZ] A. Koldobsky And A. Zvavitch, An isomorphic version of the BusemannPetty problem for arbitrary measures, Geom. Dedicata 174 (2015), 261-277.

[KMP] H. KÖNIG, M. MeYer, A. Pajor, The isotropy constants of Schatten classes are bounded, Math. Ann. 312 (1998), 773-783.

[LR] D. G. LARMan And C. A. Rogers, The existence of a centrally symmetric convex body with central sections that are unexpectedly small, Mathematika 22 (1975), 164-175.

[Lu] E. Lutwak, Intersection bodies and dual mixed volumes, Advances in Math. 71 (1988), 232-261.

[M1] E. Milman, Dual mixed volumes and the slicing problem, Adv. Math. 207 (2006), 566-598.

[M2] E. Milman, Generalized intersection bodies, J. Funct. Anal. 240 (2006), 530-567.

[M3] E. Milman, On the mean-width of isotropic convex bodies and their associated $L_{p}$-centroid bodies, preprint, arXiv:1402.0209. 
[MP] V. D. Milman And A. PaJor, Isotropic position and inertia ellipsoids and zonoids of the unit ball of a normed $n$-dimensional space, in: Geometric Aspects of Functional Analysis, ed. by J. Lindenstrauss and V. D. Milman, Lecture Notes in Mathematics 1376, Springer, Heidelberg, 1989, pp. 64-104.

$[\mathrm{Pa}]$ G. PAouris, On the isotropic constant of non-symmetric convex bodies, Geom. Aspects of Funct. Analysis. Israel Seminar 1996-2000, Lect. Notes in Math., 1745 (2000), 239-244.

[P] M. Papadimitrakis, On the Busemann-Petty problem about convex, centrally symmetric bodies in $\mathbb{R}^{n}$, Mathematika 39 (1992), 258-266

[Pe] C. M. PetTy, Projection bodies, Proc. Coll. Convexity (Copenhagen 1965), Kobenhavns Univ. Mat. Inst., 234-241.

[RZ] B. Rubin and Gaoyong Zhang, Generalizations of the Busemann-Petty problem for sections of convex bodies, J. Funct. Anal. 213 (2004), 473-501.

[Sch] R. Schneider, Zu einem problem von Shephard über die projektionen konvexer Körper, Math. Z. 101 (1967), 71-82.

[Sh] G. C. Shephard, Shadow systems of convex bodies, Israel J. Math. 2 (1964), 229-306.

[Z1] Gaoyong Zhang, Intersection bodies and Busemann-Petty inequalities in $\mathbb{R}^{4}$, Annals of Math. 140 (1994), 331-346.

[Z2] Gaoyong Zhang, A positive answer to the Busemann-Petty problem in four dimensions, Annals of Math. 149 (1999), 535-543.

[Z3] Gaoyong Zhang, Sections of convex bodies, Amer. J. Math. 118 (1996), 319-340.

[Zv] A. Zvavitch, The Busemann-Petty problem for arbitrary measures, Math. Ann. 331 (2005), 867-887.

Department of Mathematics, University of Missouri, Columbia, MO 65211

E-mail address: koldobskiya@missouri.edu 